IRA-International Journal of Technology \& Engineering

ISSN 2455-4480; Vol.03, Issue 03 (2016)

Institute of Research Advances

http://research-advances.org/index.php/IRAJTE

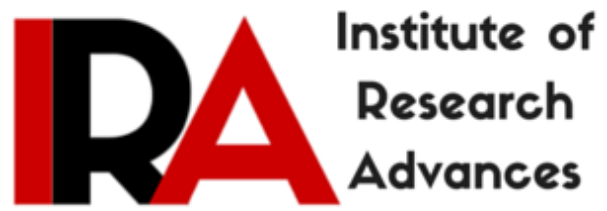

\title{
Tamil HandWritten Word Recognition with Entrenched Attributes using k- Means Algorithm
}

Aravinda C.V

Research Scholar,

VTU, Belagavi, India.

Prakash H. N

Prof \& Head Dept of CSE,

RIT, Hassan, India.

DOI: http://dx.doi.org/10.21013/jte.v3.n3.p3

\section{How to cite this paper:}

C.V, A., \& H. N, P. (2016). Tamil HandWritten Word Recognition with Entrenched Attributes using k-Means Algorithm. IRA-International Journal of Technology \& Engineering (ISSN 2455-4480), 3(3). doi:http://dx.doi.org/10.21013/jte.v3.n3.p3

(C) Institute of Research Advances

(cc) EY-NC

This works is licensed under a Creative Commons Attribution-Non Commercial 4.0 International License subject to proper citation to the publication source of the work.

Disclaimer: The scholarly papers as reviewed and published by the Institute of Research Advances (IRA) are the views and opinions of their respective authors and are not the views or opinions of the IRA. The IRA disclaims of any harm or loss caused due to the published content to any party. 


\begin{abstract}
In spite, couple types of progress in advancements identifying with Tamil Optical character affirmation, handwriting continues hanging on as strategy for reporting information for ordinary life. The methodology of division and affirmation positions quiets a huge amount of troubles especially in seeing cursive physically composed scripts of different lingos. The thought proposed is an answer made to perform tamil character affirmation of composed by hand scripts in Tamil, a language having official status in India, Sri Lanka, and Singapore. The approach utilizes KNN Algorithm technique for seeing charted off cursive decrypted Tamil characters. The conflict of the structure is obvious as it can overwhelm the complexities develop out of content style assortments and winds up being versatile and solid. More elevated amount of precision in results has been obtained with the use of this procedure on a sweeping database and the precision of the results displays its application on business use. The methodology certifications to demonstrate an essential and brisk system to create a full OCR structure connected with sensible pre-get ready.
\end{abstract}

Keywords_-Euclidean distance, Similarity, Multiple View Point

\title{
I. INTRODUCTION
}

Character acknowledgment can illuminate more mind boggling issues and facilitate the drudgery required in keeping up dark picture records. Essentially changing over checked pictures into content report can empower control through word preparing applications. Optical Character Recognition has picked up an energy since the requirement for digitizing or changing over checked pictures of machine printed or manually written content (numerals, letters, and images), in to an arrangement perceived by PCs, (for example, ASCII). OCR has been broadly utilized as the fundamental use of various learning techniques in machine learning writing [1]. Penmanship acknowledgment is the assignment of changing a dialect re-exhibited in its own particular spatial type of graphical imprints into a typical representation [2]. Penmanship acknowledgment acquired various innovations from optical character acknowledgment (OCR). The principle con-trast amongst transcribed and typewritten characters is in the varieties that accompany penmanship. It is additionally worth seeing that OCR manages logged off acknowledgment while penmanship acknowledgment might be required for both on-line and disconnected from the net signs. Penmanship acknowledgment is one of the exact testing issues. Customarily the field of penmanship acknowledgment is partitioned into logged off and on-line acknowledgment [2]. In disconnected from the net acknowledgment, as it were the picture of the penmanship is accessible for the PC, while in the on-line case transient data, for example, pentip facilitates as a capacity of time is likewise accessible. Commonplace information securing gadgets for logged off and online acknowledgment are scanners and digitizing tablets, individually. Because of the absence of fleeting data, disconnected from the net penmanship acknowledgment is viewed as more troublesome than on-line. Moreover, it is additionally clear that the logged off case is the one that compares to the customary perusing errand performed by people [3]. The requirement for OCR emerges with regards to digitizing Tamil records from the antiquated and old time to the most recent, which helps in sharing the information through the Internet [5]. Tamil, the local dialect of a southern state in India has a few million speakers over the world and is an official dialect in nations, for 
example, Sri Lanka, Malaysia and Singapore. The infiltration of Information Innovation (IT) gets to be harder in a nation such as India where the greater part individuals read and write in their local dialect. In this manner, empowering collaboration with PCs in the local dialect what's more, actually, for example, penmanship is totally vital [4].Tamil is the foundation of all Dravidian dialects like Malayalam, Telugu, Kanadam and so on, basically the native language of the Tamil individuals and talked transcendently by the general population of Tamilnadu. The dialect has increased authority status in India, Srilanka, and Singapore. Significant rate of minorities in Malaysia, Mauritius, and Reunion, as well as exiled person groups the world over moreover communicates in Tamil [20]. Tamil is the primary dialect to be given as an established dialect by the administration of India in 2004, trailed by Sanskrit [21], [22]. The dialect is additionally the managerial dialect of the Indian condition of Tamilnadu. Comprising images for vowels and consonants, Tamil scripts have a place with the group of syllabic letter sets [4]. Utilizing extraordinary diacritical imprints known as mantras, the vowels verifiably present in the consonants can be adjusted to different vowels. The certain vowel sound can be killed in a consonant by changing to its half structure utilizing the vowel-quieting diacritic. Alluded here as a syllabic unit, a composite character, is gotten by joining a vowel and a consonant. Despite the fact that the Tamil Alphabets don't have much of cursive nature, the cursive nature appears to exist in the velocity composing, composing style of people, old literary works in Tamil written in OlaiChu-vadi, Stone sacred writings and so on. Counting 12 immaculate vowels and 23 unadulterated consonants the dialect comprise 156 novel images/characters. The joined whole of immaculate vowels and immaculate consonants, the 35 characters, is the fundamental character units of the script and the remaining classifications of characters are vowelconsonant blends. The essential character and the modifier image comparing to the fundamental character are the two sections that fundamentally shape the vowel-consonant mix.

\section{RESEARCH WORK CARRIED OUT EARLIER}

Yann Lecun et al. [1] talked about different techniques connected to transcribed character acknowledgment and analyze them on a standard manually written digit acknowledgment assignment and depicted two frameworks for internet penman-ship acknowledgment that investigations show the upside of worldwide preparing, and the adaptability of chart transformer systems. Rejean Plamondon and Sargur N.Srihari [2] depicts the way of written by hand dialect, how it is transduced into electronic information and the fundamental ideas driving com-posed dialect acknowledgment calculations and they show that calculations for preprocessing, character and word acknowledgment, and execution with pragmatic frameworks. Tam'as Varga [3] research the era and utilization of manufactured preparing information as far as the issue of disconnected from the net cursive written by hand content line acknowledgment and they look at whether the acknowledgment execution can be enhanced by extending the characteristic preparing set utilizing artificially created content lines, on the grounds that the programmed era of preparing information is much quicker what's more, less expensive than gathering extra human composed examples. Bharath An and Sriganesh Madhvanath [4] have proposed an information driven HMM-based online manually written word acknowledgment framework for Tamil, an In-dic script and they examined an essayist autonomous online manually written Tamil word acknowledgment framework that utilizes HMMs for word displaying Seethalakshmi R. et al. [5] examines the different methodologies and systems required in the acknowledgment of Tamil content and they alludes Optical Character Acknowledgment (OCR) for the procedure of changing over printed Tamil content records into programming deciphered Unicode Tamil Text and 
their removed elements are gone to a Support Vector Machine (SVM) where the characters are arranged by Administered Learning Algorithm. Sung-Bae Cho [6] present three advanced neural system classifiers to settle complex example acknowledgment issues: different multilayer perceptron (MLP) classifier, concealed Markov model (Gee)/MLP half breed classifier, and structure versatile self-sorting out guide (SOM) classifier and their three techniques have delivered 97.35\%, 96.55\%, what's more, $96.05 \%$ of the acknowledgment rates, separately, which are superior to those of a few past techniques reported in the writing on the same database. K.H.Aparna et al. [7] gives a complete OCR framework for Tamil newsprint that incorporates the full suite of procedures from skew adjustment, binarization, division, content and non-content square arrangement, line, word and character division what's more, character acknowledgment to definite reproduction and their methodology gave sensible division results with the class of record pictures picked in the present work. Khalaf khatatneh et al. [8] proposes another method helps with building up an acknowledgment framework for taking care of the Arabic Hand Written content named Arabic Hand Written Optical Character Recognition (AHOCR) that worried with acknowledgment of hand composed Alphanumeric Arabic characters and their last results show and clear up that the proposed AHOCR procedure accomplishes an amazing test precision of acknowledgment appraised up to $97 \%$ for disconnected Arabic characters and $96 \%$ for Arabic content. H.Bunke et al. [10] have built up a strategy for the disconnected from the net acknowledgment of cursive penmanship based on Hidden Markov Models and their letter models are in the blink of an eye obtuse to their encompassing connections, and no endeavors have been no made to fabricate singular models for essentially distinctive acknowledge of the same letter.

\section{EXISTING TECHNIQUES}

This work exhibits an Offline Cursive Word Recognition System managing single essayist tests. The framework depends on a consistent thickness Hidden Markov Model pre-pared utilizing either the crude information, or information changed utilizing Principal Component Analysis or Independent Component Analysis. Both procedures essentially enhanced the acknowledgment rate of the framework. Preprocessing, standardization and highlight extraction are portrayed and also the preparation method embraced. A few trials were performed utilizing a freely accessible database. The precision acquired is the most elevated introduced in the writing over the same information. The framework depends on a sliding window approach: a window shifts section by segment over the picture and, at every progression, secludes an edge. A component vector is extricated from every edge and the grouping of edges so acquired is displayed with Continuous Density Hidden Markov Models (HMMs). The utilization of the sliding window approach has the vital point of preference of keeping away from the need of an autonomous division, a troublesome and blunder inclined procedure. Keeping in mind the end goal to decrease the quantity of parameters in the HMMs, we utilize corner to corner covariance grids in the emanation probabilities. This relates to the unreasonable presumption of having decorrelated highlight vectors. Consequently, we connected Principal Component Analysis (PCA) and Independent Component Analysis (ICA) to decorrelate the information. This permitted a critical change of the acknowledgment rate. The acknowledgment precision accomplished with the methodology proposed here is, to our insight, the most noteworthy among the outcomes over the same information displayed in the writing. The investigation of the acknowledgment as an element of the word length demonstrates that the framework accomplishes an acknowledgment rate for tests longer than six letters. This proposes the 
execution of our framework in errands including words with high normal length can be great. Both PCA and ICA positively affected the acknowledgment rate, PCA specifically diminished the mistake rate. A further change can most likely be acquired by utilizing nonlinear or bit PCA. Such strategies regularly work superior to the direct change we used to perform PCA. The utilization of information ward heuristics was maintained a strategic distance from keeping in mind the end goal to make the framework adaptable concerning a change of essayist. Any specially appointed calculation for the particular style of the author was maintained a strategic distance from. The earlier data about the word recurrence and appropriation can be helpful to enhance the acknowledgment of short words. These are normally articles, conjunctions and recommendations that show up frequently in the sentences. Consequently, a conceivable future course to take after is the use of dialect models that consider this sort of data.

\section{DRAW BACK OF EXISTING TECHNIQUES}

1) Keeping in mind the end goal to decrease the quantity of parameters in the HMMs, we utilize corner to corner covariance frameworks in the emanation probabilities. This relates to the unlikely presumption of having decorrelated highlight vectors.

2) Remembering the final objective to diminish the amount of parameters in the HMMs, we use corner to corner covariance structures in the transmission probabilities. This identifies with the impossible assumption of having decorrelated highlight vectors.

3) It is imperative to parcel the words into letters to perform the planning and information incident may happens.

\section{PROPOSED TECHNIQUES}

In the proposed framework, this is accomplished by a blend of name inserting and properties learning, and a typical subspace relapse. At that point the pictures and strings speak to the same word which are near each other permitting one to cast acknowledgment and recovery assignments. Contrasted and the current technique, the benefit of our strategy has an altered length, low dimensional and quick to figure. Word spotting in archive pictures has pulled in consideration in the record investigation and still stances bunches of difficulties because of the troubles of authentic reports, diverse scripts, clamor, manually written records, and so on. With respect to acknowledgment, written by hand acknowledgment still represents an imperative test for the same reasons. A model is initially prepared utilizing marked preparing information. At test time, given a picture word and a content word, the model figures the likelihood of that content word being created by the model when sustained with the picture word. Acknowledgment can then be tended to by figuring the probabilities of all the vocabulary words given the question picture and recovering the closest neighbor. As in the word spotting case, the fundamental disadvantage here is the examination speed, since figuring these probabilities is requests of greatness slower than processing an Euclidean separation or a speck item between vectorial representations. The expanding enthusiasm for extricating literary data. The high diserse nature of these photos when diverged from chronicles, basically due the far reaching appearance variability, makes it outstandingly difficult to apply routine frameworks of the record examination field. Regardless, with the late progression of skilled PC vision strategies some new systems have been proposed. To make sense of how to recuperate and see words that have not Been seen in the midst of 
setting it up, is imperative to have the ability to trade learning between the readiness and testing tests. A champion amongst the most popular approaches to manage perform this zero shot learning in PC vision incorporates the use of visual characteristics For our circumstance, we propose a more broad structure since we don't urge the choice of parts or the system to take in the qualities.

\section{METHODOLOGY}

In the first place, the picture is stacked as the information picture. Separating operations happens for the info picture. The middle channel is utilized for the expulsion of clamor and smoothening the picture. The middle channel is a non-linear computerized sifting system, regularly used to evacuate clamor. Such commotion lessening is a normal pre-handling venture to enhance the consequences of later preparing (for instance, edge location on a picture). Middle separating is broadly utilized as a part of computerized picture handling on the grounds that, under certain conditions, it jam edges while evacuating clamor. Middle separating smoothes the picture and is hence valuable in lessening clamor. Dissimilar to low pass sifting, middle separating can protect discontinuities in a stage work and can smooth a couple of pixels whose qualities vary essentially from their surroundings without influencing alternate pixels. It is likewise helpful in safeguarding edges in a picture while decreasing arbitrary commotion. Imprudent or salt-and pepper clamor can happen because of an irregular piece blunder in a correspondence channel.

\section{SEGMENTATION}

We consider two issues identified with content comprehension: word spotting and word acknowledgment. In word recognizing, the objective is to discover all occasions of a question word in a dataset of pictures. The question word might be a content string in which case it is normally alluded to as inquiry by string (QBS) or question by content (QBT) , or may likewise be a picture, in which case it is generally alluded to as question by illustration (QBE). In word acknowledgment, the objective is to get a translation of the question word picture. By and large, including this work, it is accepted that a content word reference or vocabulary is supplied at test time, and that exclusive words from that dictionary can be utilized as competitor interpretations as a part of the acknowledgment undertaking. In this work we will likewise expect that the area of the words in the pictures is given, i.e., we have entry to pictures of trimmed words. On the off chance that those were not accessible, content confinement and division strategies could be utilized. In the division procedure we are editing the words indistinguishably and show it in the jumping box.

\section{FEATURE EXTRACTION}

Gabor wavelets in picture handling calculations, in particular the interest point identification. There are a few ways to deal with the interest point identification utilizing Gabor capacities or wavelets. All the more particularly, the two most normal methodologies include the edge identification from the element picture or the corner recognition utilizing a mix of reactions to a few channels with an alternate introduction.

In this paper, another technique taking into account the Gabor wavelets is proposed. This methodology contrasts from past methodologies primarily in the way the channel reaction is figured. All the more particularly, the channel reaction is resolved just in two opposite bearings. The way of this methodology comprises in the utilization of reactions to Gabor wavelets as the halfway subsidiaries in the understood locators (e.g., Canny edge finder, Harris corner identifier, Hessian-based blob indicator). Such a methodology 
might be valuable when a quick execution of the Gabor change is accessible, or when the change is as of now precomputed. My fundamental commitment comprises of the usage of the Gabor wavelet as a multiscale incomplete differential administrator.

\section{CLASSIFICATION}

The order for this procedure is finished by utilizing KNN Classifier. Arrangement (speculation) utilizing an example based classifier can be a straightforward matter of finding the closest neighbor in case space and naming the obscure occasion with the same class name as that of the found (known) neighbour. This methodology is regularly alluded to as a closest neighbor classifier. The drawback of this straightforward methodology is the absence of strength that describes the subsequent classifiers. The high level of nearby affectability makes closest neighbor classifiers exceptionally powerless in the preparation information.

\section{K-NN TECHNIQUE ADOPTED}

Gabor wavelets in picture taking care of computations, specifically the interest point ID. There are a couple approaches to manage the interest point recognizable proof using Gabor limits or wavelets. More especially, the two most typical philosophies incorporate the edge ID from the component picture or the corner acknowledgment using a blend of responses to a couple channels with a substitute presentation. In this paper, another system considering the Gabor wavelets is proposed. This technique contrasts from past procedures principally in the way the channel response is figured. More especially, the channel response is determined just in two inverse direction. The method for this system includes in the usage of responses to Gabor wavelets as the midway auxiliaries in the comprehended locators (e.g., Canny edge discoverer, Harris corner identifier, Hessianbased blob marker). Such a procedure may be important when a fast execution of the Gabor change is available, or when the change is starting now precomputed. My key responsibility involves the use of the Gabor wavelet as a multiscale deficient differential executive. Dissimilar to numerous counterfeit learners, case based learners don't extract any data from the preparation information amid the learning stage. Learning is just an issue of epitomizing the preparation information. The procedure of speculation is delayed until it is completely unavoidable, that is, at the season of arrangement. This property has lead to the alluding to case based learners as languid learners, while classifiers, for example, bolster forward neural systems, where appropriate deliberation is done amid the learning stage, regularly are entitled anxious learners.

\section{CONCLUSION AND FUTURE ENHANCEMENT}

This paper proposes an approach to manage address and consider word pictures, both on record and on normal zones. We demonstrate how an attributes build approach based as for a pyramidal histogram of characters can be used to make sense of how to embed the word pictures and their printed elucidations into a common, more discriminative space, where the likeness between words is free of the composed work and content style, edification, get edge, et cetera. This credits representation prompts a bound together representation of word pictures and strings, achieving a strategy that licenses one to perform either request by-delineation or inquiry by-string looks for, furthermore picture interpretation, in a united structure. We test our procedure in four open datasets of reports and trademark pictures, beating best in class approachs and showing that the proposed characteristic based representation is wellsuited for word looks, whether they are pictures 
or strings, in interpreted and ordinary pictures. The eventual outcomes of our philosophy on the word spotting undertaking.

\section{REFERENCES}

[1]J. Almazan, A. Gordo, A. Fornes, and E. Valveny, Handwritten word spotting with corrected attributes, in Proc. IEEE Int. Conf. Comput. Vis., 2013, pp. 10171024.

[2] R. Manmatha and J. Rothfeder, A scale space approach for automatically segmenting words from historical handwritten documents, IEEE Trans. Pattern Anal. Mach. Intell., vol. 27, no. 8, pp. 12121225, Aug. 2005.

[3]L. Neumann and J. Matas, Real-time scene text localization and recognition, in Proc. IEEE Conf. Comput. Vis. Pattern Recog., 2012, pp. 35383545.

[4]L. Neumann and J. Matas, Scene text localization and recognition with oriented stroke detection, in Proc. IEEE Int. Conf. Comput. Vis., 2013, pp. 97104.

[5]A. Bissacco, M. Cummins, Y. Netzer, and H. Neven, PhotoOCR: Reading text in uncontrolled conditions, in Proc. IEEE Int. Conf. Comput. Vis., 2013, pp. 785792.

[6]A. Fischer, A. Keller, V. Frinken, and H. Bunke, HMM-based word spotting in handwritten documents using subword models, in Proc. 20th Int. Conf. Pattern Recog., 2010, pp. 34163419.

[7] V. Frinken, A. Fischer, R. Manmatha, and H. Bunke, A novel word spotting method based on recurrent neural networks, IEEE Trans. Pattern Anal. Mach. Intell., vol. 34, no. 2, pp. 211224, Feb. 2012.

[8] R. Manmatha, C. Han, and E. M. Riseman, Word spotting: A new approach to indexing handwriting, in Proc. IEEE Comput. Soc. Conf. Comput. Vis. Pattern Recog., 1996, pp. 631637.

[9] T. Rath, R. Manmatha, and V. Lavrenko, A search engine for historical manuscript images, in Proc. 27th Annu. Int. ACM SIGIR Conf. Res. Develop. Inform. Retrieval, 2004, pp. 369376.

[10] T. Rath and R. Manmatha, Word spotting for historical documents, Int. J. Document Anal. Recog., vol. 9, pp. 139152, 2007.

[11] J. A. Rodrguez-Serrano and F. Perronnin, Local gradient histogram features for word spotting in unconstrained handwritten documents, presented at the Int. Conf. Frontiers Handwriting Recognition, Montreal, QC, Canada, 2008.

[12] J. A. Rodrguez-Serrano and F. Perronnin, A model-based sequence similarity with application to handwritten wordspotting, IEEE Trans. Pattern Anal. Mach. Intell., vol. 34, no. 11, pp. 21082120, Nov. 2012.

[13] S. Espana-Bosquera, M. Castro-Bleda, J. Gorbe-Moya, and F. Zamora-Martinez, Improving offline handwritten text recognition with hybrid HMM/ANN models, IEEE Trans. Pattern Anal. Mach. Intell., vol. 33, no. 4, pp. 767779, Apr. 2011. 
[14] I. Yalniz and R. Manmatha, An efficient framework for searching text in noisy documents, in Proc. 10th IAPR Int. Workshop Document Anal. Syst., 2012, pp. 4852.

[15] K. Wang, B. Babenko, and S. Belongie, End-to-end scene text recogni-tion, in Proc. IEEE Int. Conf. Comput. Vis., 2011, pp. 14571464.

[16] A. Mishra, K. Alahari, and C. V. Jawahar, Top-down and bottomup cues for scene text recognition, in Proc. IEEE Conf. Comput. Vis. Pattern Recog., 2012, pp. 26872694.

[17] J. A. Rodrguez-Serrano and F. Perronnin, Label embedding for text recognition, in Proc. Brit. Mach. Vis. Conf., 2013, pp. 5.15.12.

[18] C. Leslie, E. Eskin, and W. Noble, The spectrum kernel: A string kernel for SVM protein classification, in Pacific Symp. Biocomput., 2002, pp. 564575.

[19] H. Lodhi, C. Saunders, J. Shawe-Taylor, N. Cristianini, and C. Watkins, Text classification using string kernels, J. Mach. Learn. Res., vol. 2, pp. 419444, 2002.

[20] H. Jegou, M. Douze, and C. Schmid, Product quantization for nearest neighbor search, IEEE Trans. Pattern Anal. Mach. Intell., vol. 33, no. 1, pp. 117128, Jan. 2011.

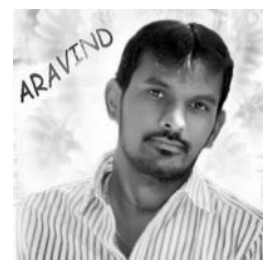

Author 1- Aravinda cv currently working as Asst Prof at SJBIT college, kengeri, Bengaluru, Karnataka India. He has published 8 journals 7 Intenational papers and 3 National Level Conference papers.He has got 9 years of Teaching Experience at various Engineering Colleges. Currently he is pursuing his PhD at VTU Belgavi.

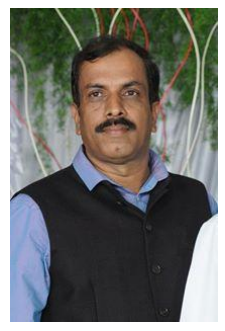

Author 2- Dr Prakash H.N Currently Professor and Head, Dept of Computer Science and Engineering Department at Rajeev Institute of Technology Hassan. He Completed his Ph.D (Computer Science) at Mysore University, M.Tech from N.I.T Warrangal, Hyderbad , B.E. at P.E.S(Mandya).He has got 24 years of Teaching in Engineering College. He has published 25 International Papers, 15 Journals and 15 National Level Conference papers. His area of interest Digitial Image Processing,Character Recog-nition,Signal Systems. 


\section{RESUTLS TESTED WITH SOME SAMPLES}

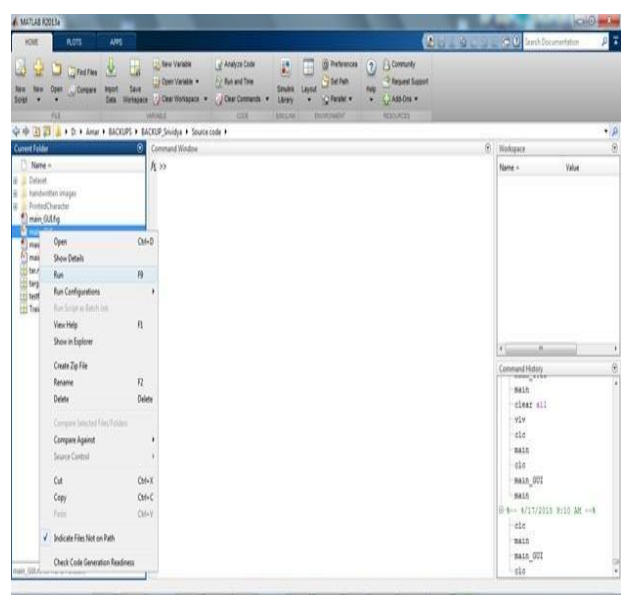

Fig. 1. Outline of Matlab Program

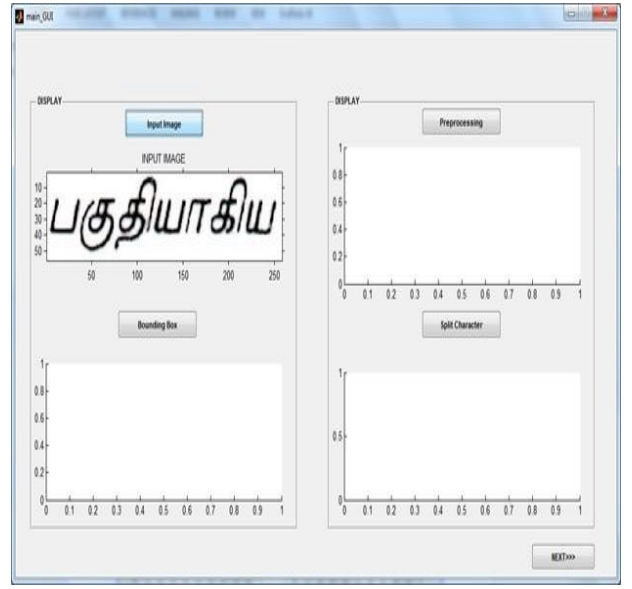

Fig. 2. Input of Tamil Word Sample

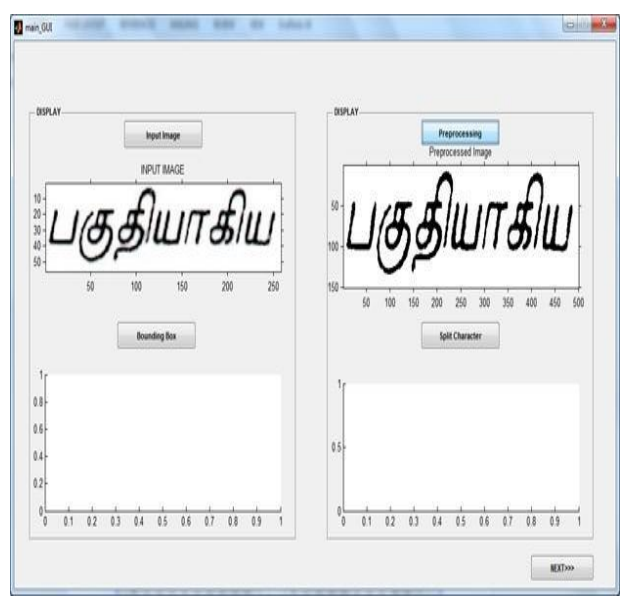

Fig. 3. Binary Format

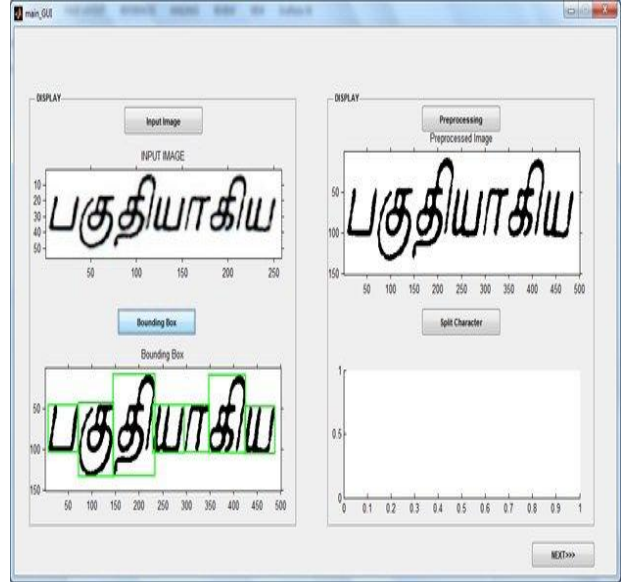

Fig. 4. Boundary Box Detection

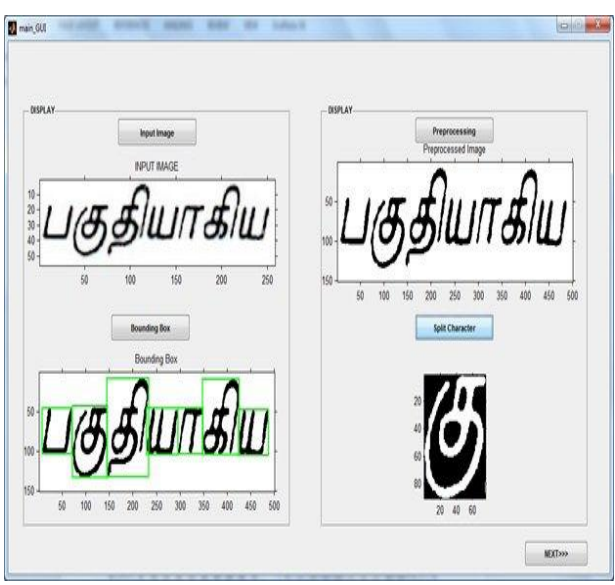

Fig. 5. Segmentation of Tamil Word Sample

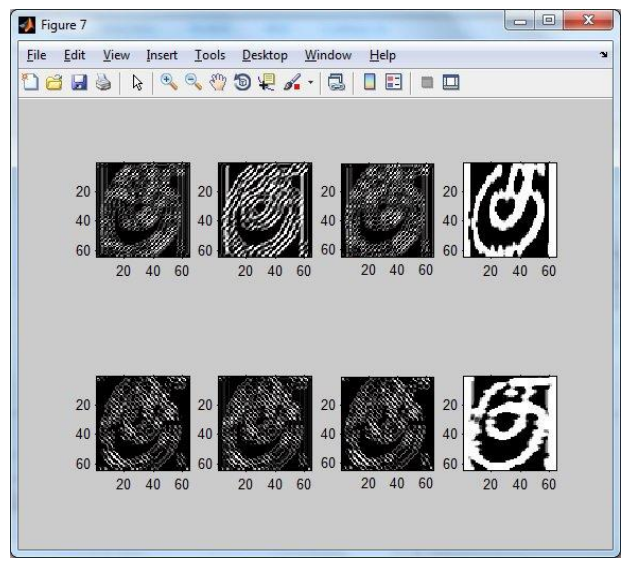

Fig. 6. Feature Extraction of Tamil Word Sample 


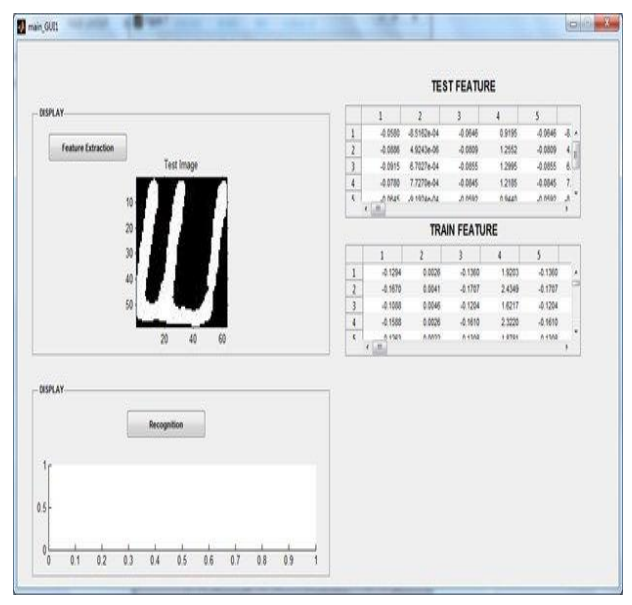

Fig. 7. Feature Extracted Values of Tamil Word Sample

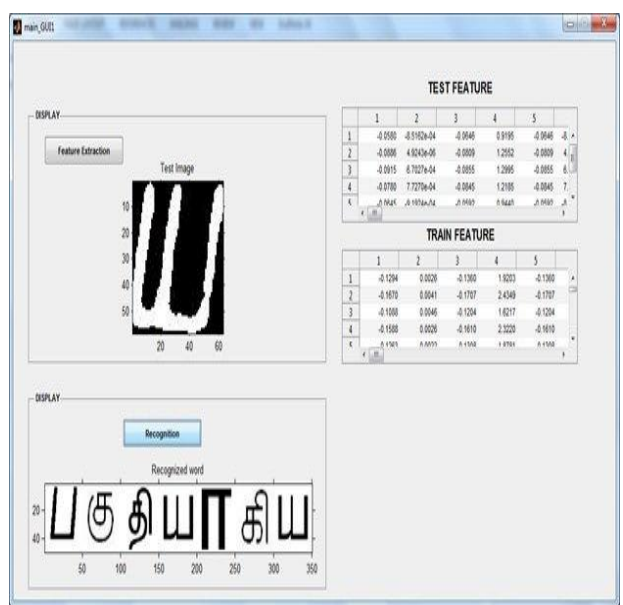

Fig. 8. Recognized output of Tamil Word Sample 